\title{
A CROSS-CORRELATION TECHNIQUE FOR RELOCATION OF SEISMICITY IN THE WESTERN CORINTH RIFT
}

\author{
Kapetanidis V. ${ }^{1}$, Papadimitriou P. ${ }^{1}$ and Makropoulos K. ${ }^{1}$ \\ ${ }^{1}$ National and Kapodistrian University of Athens, Faculty of Geology and Geoenvironment, \\ Department of Geophysics-Geothermics, 15784 Athens, Greece,vkapetan@geol.uoa.gr, \\ ppapadim@geol.uoa.gr,kmacrop@geol.uoa.gr
}

\begin{abstract}
Local seismological networks provide data that allow the location of microearthquakes which otherwise would be dismissed due to low magnitudes and low signal-to-noise ratios of their seismic signals. The Corinth Rift Laboratory (CRL) network, installed in the western Corinth rift, has been providing digital waveform data since 2000. In this work, a semi-automatic picking technique has been applied which exploits the similarity between waveforms of events that have occurred in approximately the same area of an active fault. Similarity is measured by the crosscorrelation maxi-mum of full signals. Events with similar waveforms are grouped in multiplet clusters using the nearest-neighbour linkage algorithm. Manually located events act as masters, while automatically located events of each multiplet cluster act as slaves. By cross-correlating the $P$-wave or $S$-wave segments of a master event with the corresponding segments of each of its slave events, after appropriately aligning their offsets, the measured time-lag at the cross-correlation maximum can be subtracted from the arrival-time of the slave event. After the correction of the arrival-times, a double-difference technique is applied to the modified catalogue to further improve the locations of clusters and distinguish the active seismogenic structures in the tectonically complex Western Corinth rift.
\end{abstract}

Key words: cross-correlation, multiplets, clustering, master events, double-difference, relocation, Corinth Rift, Greece.

\section{Introduction}

The Western Corinth rift is one of the most tectonically active regions in Greece. Its continuous microseismicity is closely monitored by permanent seismological stations of the Universities of Athens and Patras as well as by the Corinth Rift Laboratory (CRL) local network (Bernard et al., 2006). The growing quantity of waveform and catalogue data makes imperative the need for automatic location algorithms with improved accuracy. Some groups of events are strongly clustered in space. When their magnitudes and focal mechanisms are similar, then their recorded waveforms on each recording station are also similar. These events are called multiplets (Poupinet et al., 1984) and can be useful for relocation procedures, which is the subject of this paper.

The primary mathematical tool for the detection of multiplets and for the extraction of information from their similar waveforms is the cross-correlation function (Schaff \& Waldhauser, 2005; Hansen et al., 2006). The characteristics of the cross-correlation function will be discussed below, 


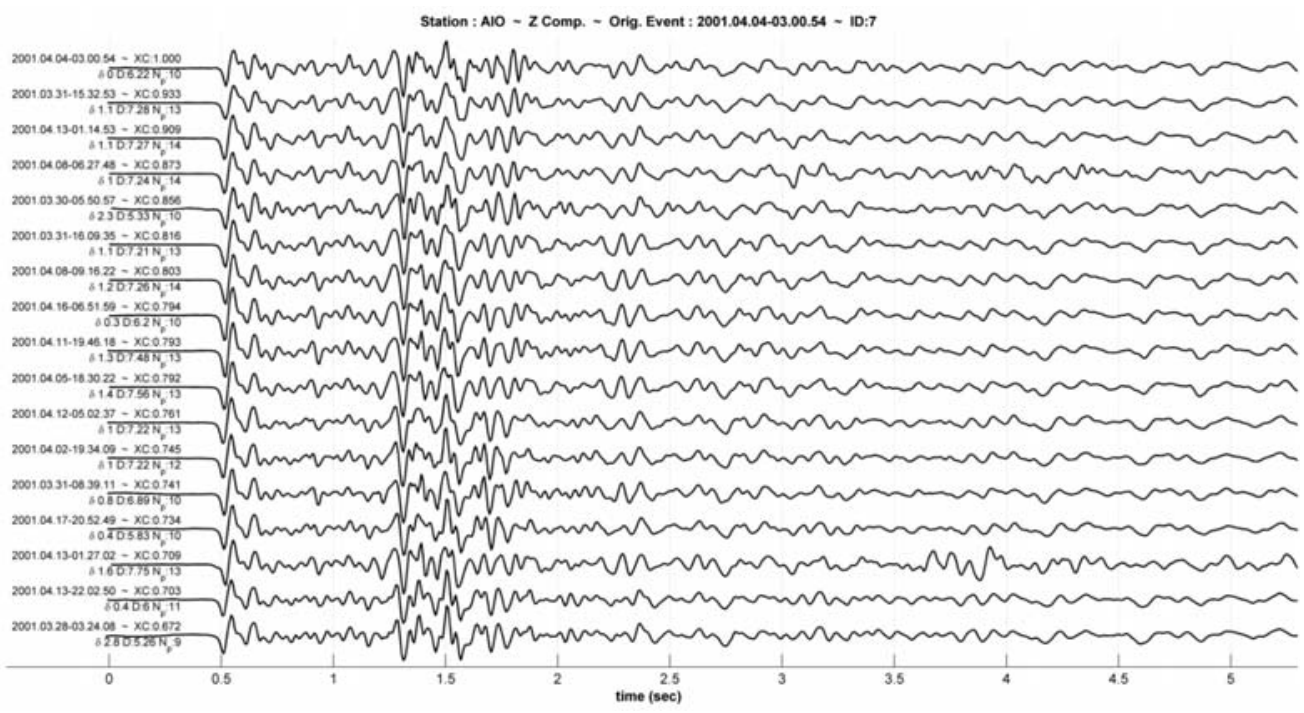

Fig. 1: vertical component waveforms of multiplets recorded at the AIO station during March-April 2001.

as well as its advantages in comparison to its spectral counterpart, the coherence function (Rubin et al., 1999; Waldhauser et al., 1999). An automated procedure will be run on several groups of earthquakes to produce cross-correlation matrices that will reveal the existence of multiplets, which will then be grouped in clusters by applying a nearest-neighbour linkage on the matrix.

A semi-automatic procedure to improve the locations of events will also be demonstrated. This will be done by using a small amount of manually picked events from multiplet clusters as "Master Events" in order to correct or add more P or S-wave arrival-times to the other multiplets ("Slave Events") of the same cluster, thus 1) improving the database without having to manually pick the arrival-times for all these events, 2) being able to add arrival-times correctly even if the signal-to-noise ratio is low.

Finally, the multiplets will be relocated with a double-difference algorithm (Waldhauser \& Ellsworth, 2000) using both catalogue and waveform cross-correlation differential travel-time data to improve the location of the multiplets' hypocenters. This has been applied to a selected group of events during 2000-2001 and 2005-2006, for the purposes of the present work, before applying it to the whole time period.

\section{Waveform Similarity}

It is expected by the theory of elastic wave propagation through layered media (Aki \& Richards, 1980) that earthquakes with similar focal parameters should produce similar waveforms. By reversing the argument, when two events have similar waveforms it is expected that they also have similar focal parameters. This is true when the properties of the medium are also similar, so that the ray-paths don't change significantly.

A pair of such events is called doublet. A cluster of more than 2 similar events is called multiplet (Fig. 1 and 6c). In the present paper the similarity between the focal parameters of two events is estimated by the degree of similarity between their full waveforms, containing P- 
(a)

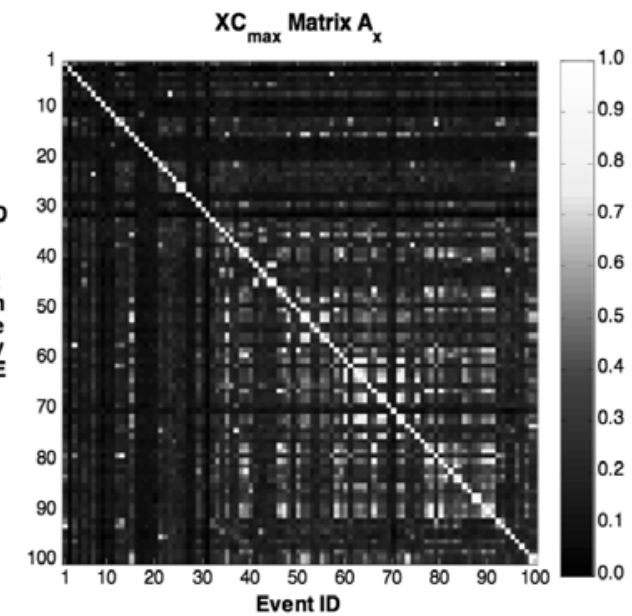

(b)

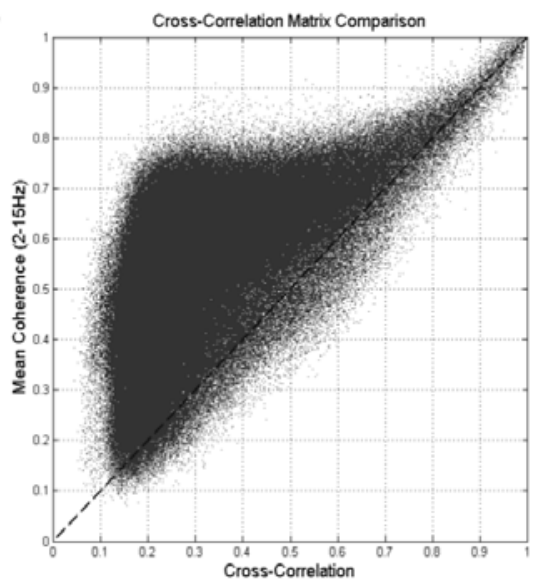

Fig. 2: (a) Cross-correlation matrix for the first 100 events of Group $A_{s}$; (b) element-by-element comparison between cross-correlation $\left(X C_{\text {max }}\right.$, horizontal axis) and mean coherence ( $m_{c o h}$, vertical axis) matrices for Group $A_{s}$.

waves, $\mathrm{S}$-waves and coda-waves. In the case of local microearthquakes it has been observed that recordings of a single vertical component from a single station is usually enough to distinguish different groups of multiplets with strong similarity between events of a single cluster and apparent dissimilarity between events of different, although neighbouring clusters, allowing for a detailed distinction of groups of events with different properties within a larger cluster.

The quantification of similarity between a pair of waveforms $x, y$ has been done in two ways: a) by cross-correlation in the time-domain, and b) by spectral coherence in the frequency-domain. The cross-correlation function between two waveforms has a positive maximum value, $X C_{\text {max }}$, on a certain time-lag $\tau_{\mathrm{m}}$. When this offset is applied to the first time-series, $\mathrm{x}(\mathrm{t})$, its waveform fits best to the other time-series, $\mathrm{y}(\mathrm{t})$, provided that the $X C_{\max }$ is relatively high. If there is no correlation between the two time-series both maximum and minimum values of the cross-correlation function will be close to zero. A strong negative (close to -1) indicates that the two time-series are negatively correlated.

When dealing with absolute times, the difference between the times of the first sample of each time-series has to be taken into account. This is crucial because it allows for a calculation of the time-lag of $X C_{\max }$ for a custom initial temporal alignment of the two time-series. More specifically, the two time-series could be aligned at the arrival-time of the P-waves or S-waves. Then, if these waveforms are similar, the time-lag provides a measure of the difference that arises between the corresponding picked arrival-times, which have been used for the temporal alignment, when the similar waveforms are shifted in time to fit together.

The spectral coherence measures the similarity between the spectra of the two time-series. It is the equivalent of the cross-correlation in the frequency domain and has been preferred by several authors (Got et al., 1994; Kilb \& Rubin, 2002) or used as an additional weight (Schaff et al., 2004). In the case of local microearthquakes, the mean spectral coherence, $m_{\text {coh }}$, between $2-15 \mathrm{~Hz}$ could be used as a measure of similarity between pairs of waveforms (similar mean values of coherence have been used by Got et al., 1994;Schaff et al., 2004). This spectral region 
avoids long period as well as high frequency noise while it contains most of the information content that is generated by a local event. Concerning the time-domain, before applying the cross-correlation function, a band-pass filter is needed as well, mainly for the removal of longperiod trends which can greatly affect the results of the cross-correlation. The equivalent value of the time-lag, $\tau_{\mathrm{m}}$, in the spectral domain, which can be used as an offset for the best temporal fit of the two waveforms, can be estimated by the slope of the phase of the cross-spectrum, which is the Fourier Transform of the cross-correlation (Poupinet et al., 1984).

A comparison between the results of the two methods has been done on a sample of 1134 events (Group $A_{s}$ ) which took place between March 26th 2001 and April 18th 2001 in the Western Corinth rift. In April 8th 2001 a $\mathrm{M}_{\mathrm{L}}=4.7$ event occurred 5km south of the city of Aigion, very close to AIO station of the CRL network (Lyon-Caen et al., 2004; Janský et al., 2004; Zahradnik et al., 2004). There was some foreshock activity in the region, as well as a spatially dense series of aftershocks. AIO station provided waveform data during that period of time.

A comparison of full-waveform recordings (in a window containing P, S, and coda-waves) of the vertical component of station $\mathrm{AIO}$ has been done between all possible combinations of pairs of events of that group. The $X C_{\max }$ and $m_{c o h}$ values were calculated. This led to the construction of the corresponding 1134x1134 square matrices. An element-by-element comparison has been done between the values of the $X C_{\max }$ and $m_{c o h}$ matrices (Fig. 2b). Although there's good correlation between $X C_{\text {max }}$ and $m_{c o h}$ values above 0.85 , it is apparent that the $m_{c o h}$ values are prone to faster saturation than the corresponding $X C_{\max }$ values: the threshold can be lowered much more in the $X C_{\max }$ axis than in the $m_{c o h}$ axis until it meets the noise background. This means that there are many cases with relatively high $m_{c o h}$ for a pair of events with very low $X C_{\text {max }}$. This observation is in agreement with a similar point made by Schaff et al. (2004), who used the sum of $X C_{\max }$ with $m_{\text {coh }}$ instead of either function alone, with 1.70 threshold.

On the other hand, the value for the time-lag that corresponds to the global maximum of the cross-correlation can be directly calculated in the time-domain with an accuracy that is limited by the sampling rate $(0.008 \mathrm{sec}$ for a sampling rate of $125 \mathrm{samples} / \mathrm{sec}$, as in the case of AIO station). The error of measurement of the equivalent time-lag in the spectral domain by the slope of the cross-spectral phase derives from a weighted least-squares linear fit and is usually much lower than the sampling interval. The weights for each spectral sample are given by the corresponding coherence (Poupinet et al., 1984). In this work the temporal cross-correlation function has been used for the calculation of time-lags in order to avoid some possible instability issues which appear in the frequency-domain.

The limitation in the accuracy of the calculation of time-lag $\tau_{\mathrm{m}}$ from the cross-correlation function can be surpassed by re-sampling the cross-correlation function using non-linear spline interpolation in a region that includes the global maximum, $X C_{\max }$. Afterwards, the time-lag of the interpolation can be calculated with accuracy limited by the re-sampling rate. 1000 samples per second are usually more than enough for a good estimation of the time-lag. Similar approach has been taken by Deichmann \& Garcia-Fernandez (1992) who used a $2^{\text {nd }}$ degree polynomial interpolation (parabola) around the global maximum of the cross-correlation.

\section{Data Preparation, Clustering}

The available catalogue data for the years 2000-2001 are spatially separated in 2 groups. Group A consists of 1954 events of a swarm that took place during the time period between February- 


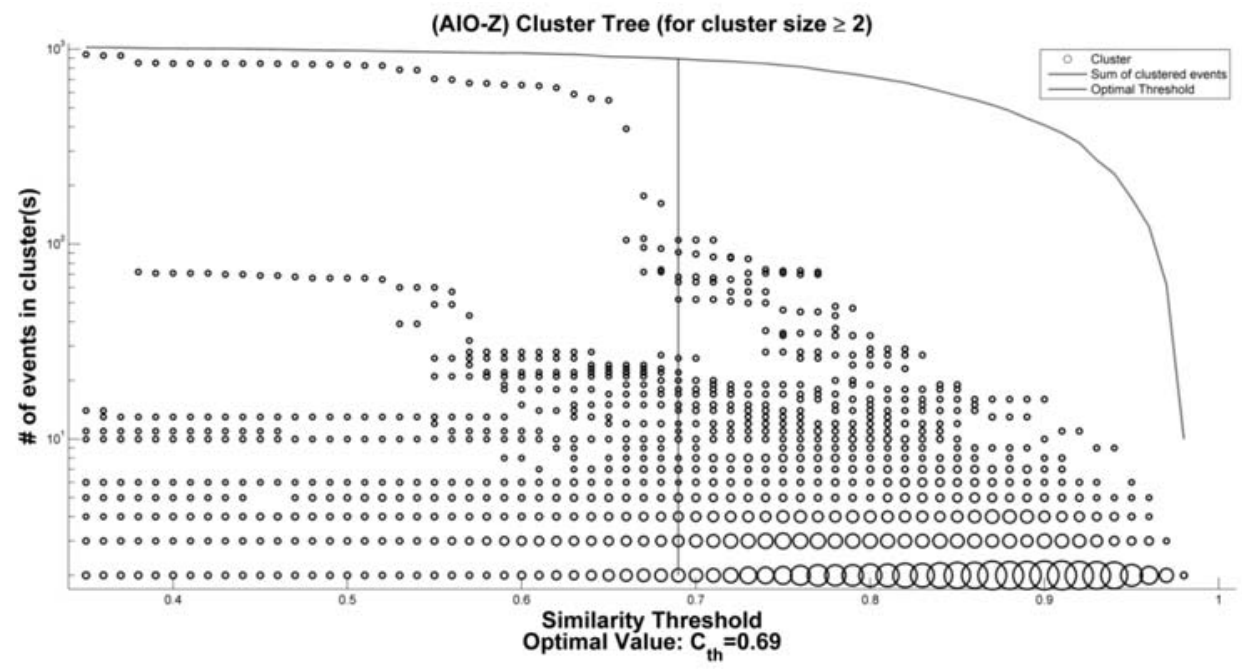

Fig. 3: Tree-diagram of multiplet clusters for Group $\mathrm{A}_{\mathrm{s}}$. Circles denote clusters formed on each similarity threshold (horizontal axis), circle size corresponds to the number of clusters containing the same number of events. The envelope curve is sum of clustered events on each similarity threshold. The vertical dashed line marks the optimal threshold $C_{t h}=0.69$.

July 2001, with epicenters close to the station AIO of the CRL network. Group B consists of 1827 events with epicenters mainly within the gulf, covering the time-period between May 2000 - December 2001. Some extra criteria that have been applied on the choice of these groups are Azimuthal Gap $<=320, N_{p h}>=8$, RMS error $<0.2 \mathrm{sec}$, Location Error $<=1.5 \mathrm{~km}$ for Group A, and Gap $<=270, N_{p h}>=5$, RMS error $<0.3 \mathrm{sec}$, Location Error $<5 \mathrm{~km}$ for Group B, where $N_{p h}$ is the number of arrival-times. The azimuthal gap limit for Group A is lower due to the fact that the epicenters of this group are at the margins of the network. For the year 2005, 1232 events were selected (Group C) with the following criteria: $N_{p h}>=9$, Location Error $<3 \mathrm{~km}$. The seismicity during that year is dispersed and there are only a few small clusters spread around the whole area. On the other hand, the seismic activity during year 2006 was increased and the spatial distribution was separated in 3 groups using a ward linkage method: groups D, E and F contain 1108,888 and 1652 events respectively.

A cross-correlation matrix is constructed for each group of earthquakes using full-waveform data on a single station and component of choice (usually the one which is closer to the group, like station AIO for Group A). The cross-correlation matrices contain information of similarity between all combinations of event-pairs within the group in a temporal sequence. The visualization of the cross-correlation matrix can provide a quick preview of the existence of temporally separated multiplet groups.

The clustering is achieved by the use of simple or nearest-neighbour linkage algorithm. For a certain threshold value $C_{t h}$, which can be modified, all rows of the matrix are scanned and the events are grouped according to the logic "if a pair $(\mathrm{A}, \mathrm{B})$ has $X C_{\max }>=C_{t h}$ and the same is true for a pair $(B, C)$, then all three events $(A, B, C)$ belong to the same cluster", or in simpler words "if A is similar to B and B is similar to $C$, then A should be similar to $C$, possibly with a different degree of similarity". A direct comparison of A with $\mathrm{C}$ may not result in a $X C_{\text {max }}>=C_{t h}$, but these events 
are connected together through B. This could be the case for events with different depths, with $\mathrm{A}$ and $\mathrm{C}$ being at the top and bottom respectively while $\mathrm{B}$ being in the middle. Pairs like A-B or B-C are called "close-relatives" while pairs like A C are called "distant-relatives".

This type of linkage can lead to wide clusters which consist of events with i.e. a) slightly different source duration but similar location of hypocenter and similar focal mechanism, or b) similar source and mechanism but slightly different hypocenter etc. Two whole sub-clusters A and $\mathrm{C}$ could be connected through a single event $\mathrm{B}$ so it is important that the threshold is chosen carefully. The number of clusters and their sizes (the number of events they contain) is also controlled by the chosen threshold value. While its choice is generally subjective, a special criterion has been used in order for one to find the "optimal threshold" value $C_{t h}$. In Fig. 3 the circles represent clusters of Group $\mathrm{A}_{\mathrm{s}}$ which are formed for the various choices of threshold value (horizontal axis) while the vertical, log-scale axis shows the size of each cluster. The envelope curve, above the circles, shows the sum of all events which are contained in clusters with size equal or larger than 2 for each threshold value. Circle size corresponds to the number of clusters of a certain cluster-size (containing the same number of events). A large number of doublets (clusters containing 2 events) is formed for high threshold values (big circles at the bottom of Fig. 3). As the threshold is lowered, more events are being clustered (the envelope curve is always increasing with the threshold decreasing). At very low thresholds, most events are grouped on what could be called the "main branch", the largest cluster that tends to accumulate all the smaller ones. In some cases there can be two or more large branches which will eventually connect once the threshold becomes low enough.

For a certain threshold value the difference between the sum of clustered events (envelope curve) and the size of the largest cluster is maximized. This is proposed as the optimal threshold value, $C_{t h}$, which is denoted by the dashed vertical line in Fig. 3. Note that the maximization of that difference is not obvious graphically as the vertical axis is in log-scale, whose purpose is to make the branching of the smaller clusters clearer.

\section{Master Events - Relocation}

For the time-span 2005-2006 the available arrival-times data have been generated by an automatic picking algorithm. Although it is able to pick both $\mathrm{P}$ and $\mathrm{S}$-wave arrival-times, like all automated processes, it has its downfalls. Some arrivals are not picked at all, while some others are erroneously picked. The resulting distribution of epicenters is dispersed with a few well-located events and many others with relatively large location errors. While the best solution to this problem is to manually pick the arrivals for all those small events, it is also time-consuming given that in 2006 alone there have been over 10000 events recorded in the Western Corinth rift by the local network. It is interesting to find out what can be achieved by applying a semi-automatic procedure that will replicate manual picks of arrival-times in sub-groups of events that have been identified as multiplets.

After applying the cross-correlation and clustering procedures, as described in the previous paragraph, a fraction of events have been grouped in multiplet clusters. One event from each cluster must be selected according to the following criteria: 1) the one with the largest number of picked arrival-times, 2) if more than one event were derived from step (1), then select the event with the smallest RMS error. These events, which will be called "Master Events", should possibly have acceptable signal-to-noise ratio in most stations and should be easily manually picked. By exploiting their similarity to the other multiplets (slaves) of the cluster to which they belong, the manually picked arrival-times will be instantiated to all the slave events, be- 

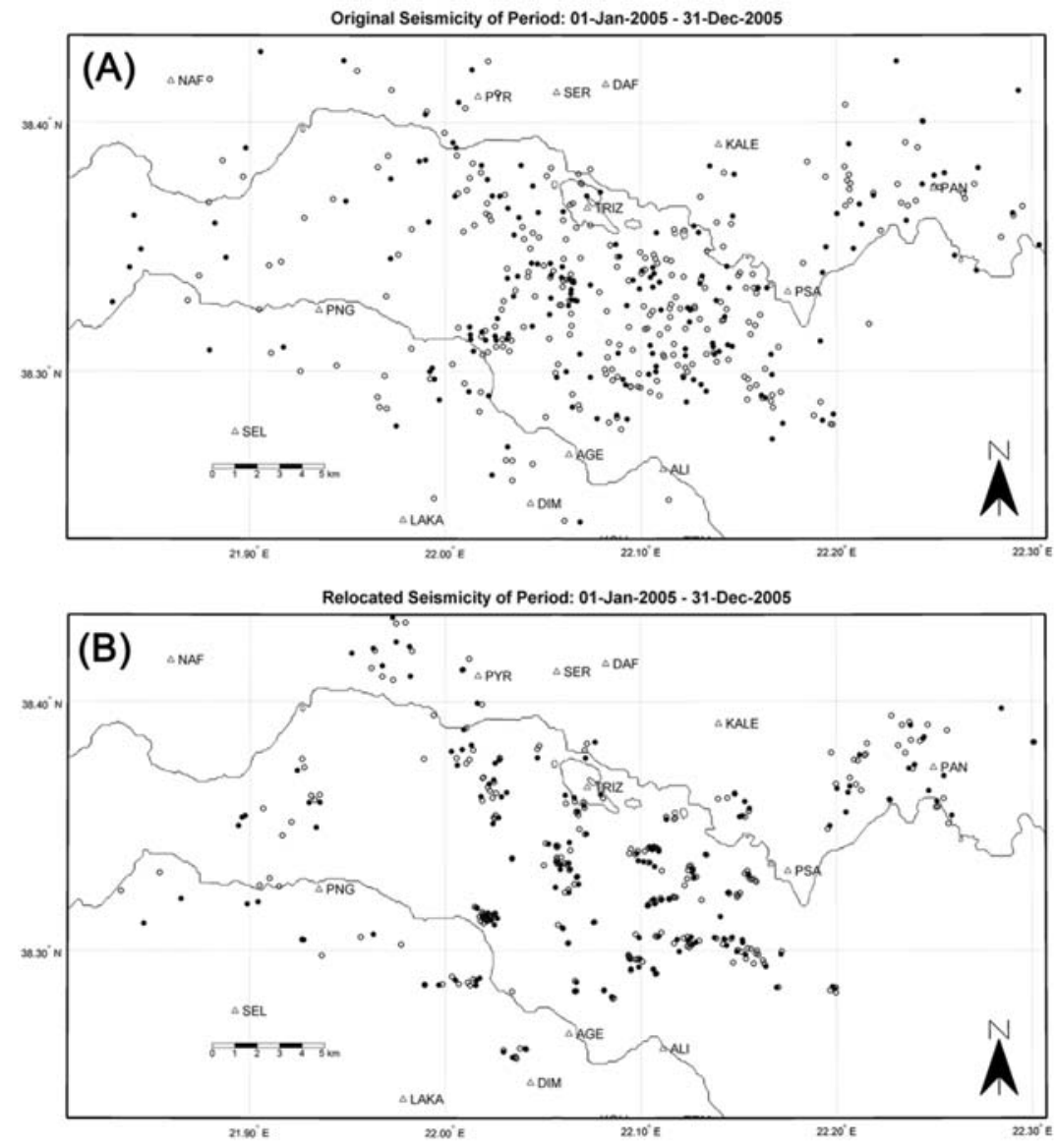

Fig. 4: (A): automatically located epicenters of multiplet clusters belonging to group C (year 2005); (B): the same events relocated using the Master Events method and HypoDD. Master Events are represented by filled circles while slave events are represented by hollow ones.

ginning with the close-relatives. Theoretical arrival-times of $\mathrm{P}$ and $\mathrm{S}$-waves at all stations need also be calculated for all slave events, in order to properly crop the corresponding waveform windows as will be described next.

For every master event the cross-correlation matrix (which has been created by using the Zcomponent of the closest station to the group of epicenters) can supply a group of close-relatives for a given $X C_{\text {max }}$ threshold. Although the optimal threshold $C_{t h}$ is advised, lower thresholds can also be used but with caution. Higher values will only result in less automatically corrected events. The P-wave part of a master event's waveform on each station/component is cropped before the $\mathrm{S}$-wave arrival. The $\mathrm{S}$-waves are cropped separately as well. Then for each slave waveform, on the same station/component, the master P-wave window slides along the time axis until the two waveforms match.

This is easily done by calculating the correlation coefficient between the amplitudes of the master/slave waveforms for each window until it reaches a maximum value that is above an ac- 

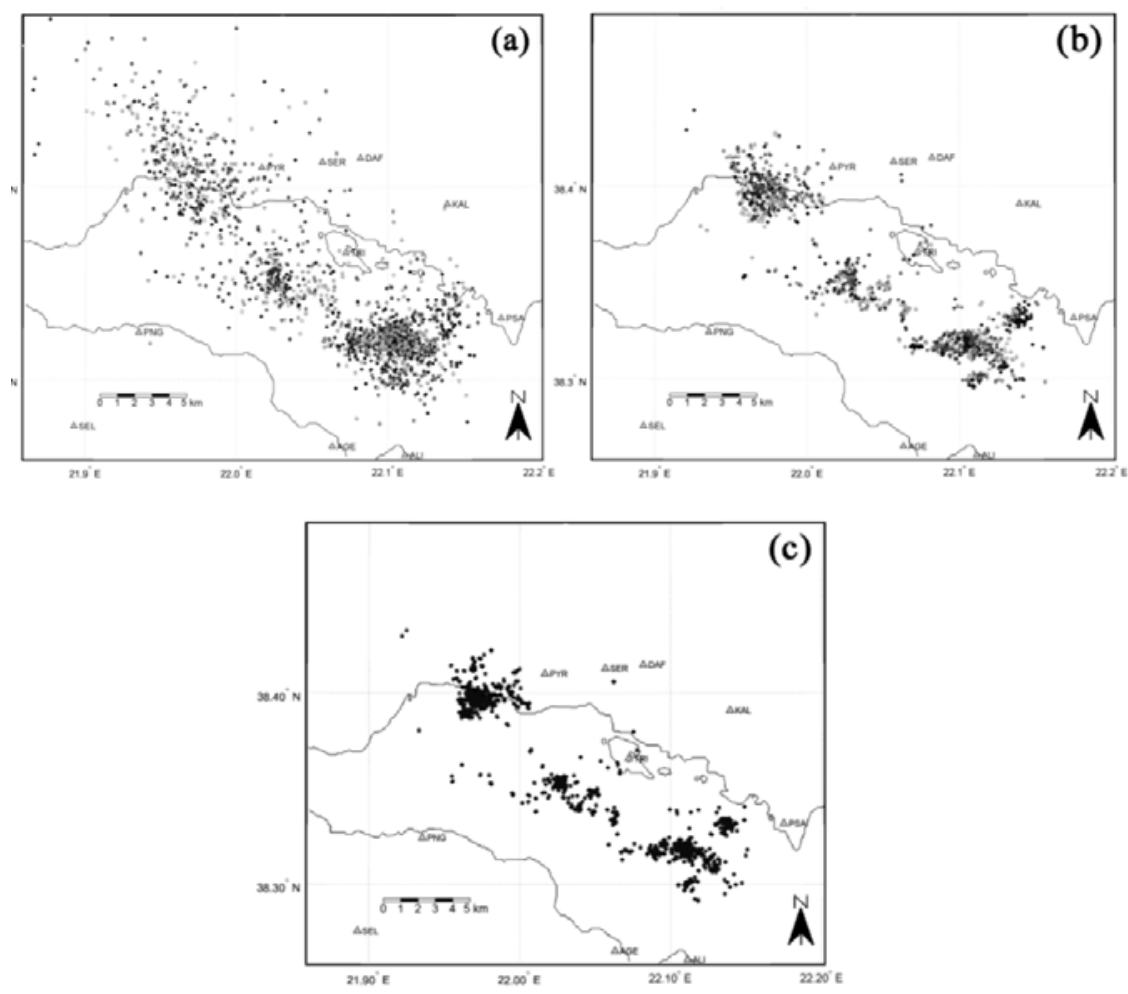

Fig. 5: (a): automatically located epicenters of multiplet clusters belonging to groups $D, E$ and $F$ (year 2006); (b): the same events relocated using the Master Events method; (c): final relocation with a double-difference technique (HypoDD).

ceptable threshold (e.g. 0.7). Both waveforms have to be band-passed prior to this procedure, in order to remove long period and high frequency noise thus allowing the strongly cohered part of their spectrum to be compared. The length of the time-series for a slave-event must extend to some seconds before the automatic (or theoretical) P-wave arrival-time and up to about 1 minute after the S-wave arrival (when one deals with small, local events). This is important in cases of arrival-times that have been largely miscalculated by the automatic picking algorithm.

Suppose a certain master event, $M$, recorded at station PSA and the time-series $M(t)$ of its cropped P-wave on the vertical component. The manually picked P-wave arrival-time for this event on this station is on $t=t_{P}$ with $t=0$ at the first sample of the series. The corresponding waveform of a slave event, $S$, is divided into a family of time-series called $S_{i}(t)$, where the index i refers to a certain sliding-window with equal length to the $M(t)$ series and which shifts by one sample each time. In $S_{i}(t)$, the relative time is $t=0$ at the first sample of each window. The correlation coefficient is calculated between $\mathrm{M}$ and $\mathrm{S}_{\mathrm{i}}$ for every $i$ until it maximizes above a threshold when $i=m$. When it does, $S_{i=m}$ and $M$ should be highly similar. Then, the absolute time of the sample at $S_{i=m}\left(t=t_{P}\right)$ should correspond to the correct arrival-time of the P-wave of the slave event on station PSA. The same process is repeated for all stations and available components and for every slave event, for both P and S-wave windows. 
Some post-processing is needed in order to evaluate the results before exporting the output files with the final arrival-times information for each slave event. A criterion for good-quality of the result is the small temporal difference of all corrections made for the arrival of a certain phase on a certain station in all available components: if they are not in good agreement this is an indication of a possible source of error. Another criterion for the quality assessment is the availability, both in the master and slave event, of the vertical-component for the P-waves and of at least one horizontal component for the S-waves. The daily status of each of the 3 components of all stations has been checked and time-periods of non-availability of certain components have been marked in a detailed table to be used whenever waveforms are used in cross-correlations.

The maximum value of the correlation coefficient, $C_{\max }$, which corresponds to the best fit between the master and slave waveform windows, should also have a strong impact on the value of the weight to be applied on the arrival-time. If its value is too low (i.e. $C_{\max }<0.5$ ) then the correction must be discarded, while for intermediate values (i.e. $0.5<C_{\max }<0.75$ ) the weight should be lowered and the use of that correction should depend on whether the other criteria are fulfilled as well.

When the first pass of corrections on arrival-times of all slave events has been completed, these corrected events can be used as master events themselves and the whole procedure is repeated. Old master events (those with the manually picked arrival-times) should not be affected by this second-level pass. By repeating the process, the corrections are applied to distant-relatives of the original master events, hence the whole cluster is corrected by using close-relatives in a master/slave hierarchy on each step, until there are no more relatives to be found. That's the main reason why the optimal $X C_{\text {max }}$ threshold, $C_{t h}$, should be used on the cross-correlation matrix as the nearest-neighbour linkage is working here as well.

Although the matrix has been created by using a single station - single component and full-waveforms, the Master Events method is using all stations and components as well as P-waves and $S$-waves separately. Events with partially similar waveforms can have different $t_{S}-t_{P}$ durations, while having similar both $\mathrm{P}$ and $\mathrm{S}$-waves. Full-waveform comparison is mostly affected by the $\mathrm{S}$-wave and coda, but dissimilarities in the $\mathrm{P}$-waves and difference in $\mathrm{t}_{\mathrm{s}}-\mathrm{t}_{\mathrm{p}}$ duration can slightly decrease the $X C_{\max }$ as well. This dissimilarity usually increases in distant-relatives within the same multiplet cluster, while it should not be noticeable in close-relatives. So, the Master Events method may replicate the manually-picked arrival-times of the master events to the slave events, but it does not strictly replicate $t_{S}-t_{P}$ time differences. That's the main reason why the hypocenters of multiplets in the final results are usually close together but not all at the exact same spot.

\section{Conclusions-Results}

The method of "Master Events" was used in several earthquake groups. In group C (year 2005) 180 master events were used to correct 328 slave events (Fig. 4). In groups D, E and F (year 2006) a total of 361 master events were used to correct 1261 slave events (Fig. 5). Followed by relocation with a double-difference method, the results are quite impressive. It is possible to expand this method to improve/add more arrival-times for events outside the previous groups. Depending on the threshold value used in such a procedure it is possible to correct 221 out of $4330(5.1 \%)$ events from year 2005 and 321 out of 4428 (7.2\%) events of 2006 by using a combination of 2130 master events (508 from year 2005 and 1622 from year 2006). If that value is lowered to $C_{t h}=0.35$ the corrected events arise to 330 (7.6\%) and 539 (12.2\%) for years 2005 and 2006 respectively. The hypocentral depths are also improved as a result of the relocation procedure. This

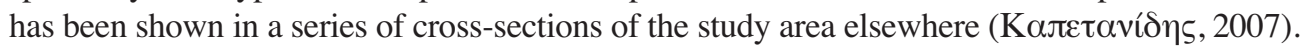



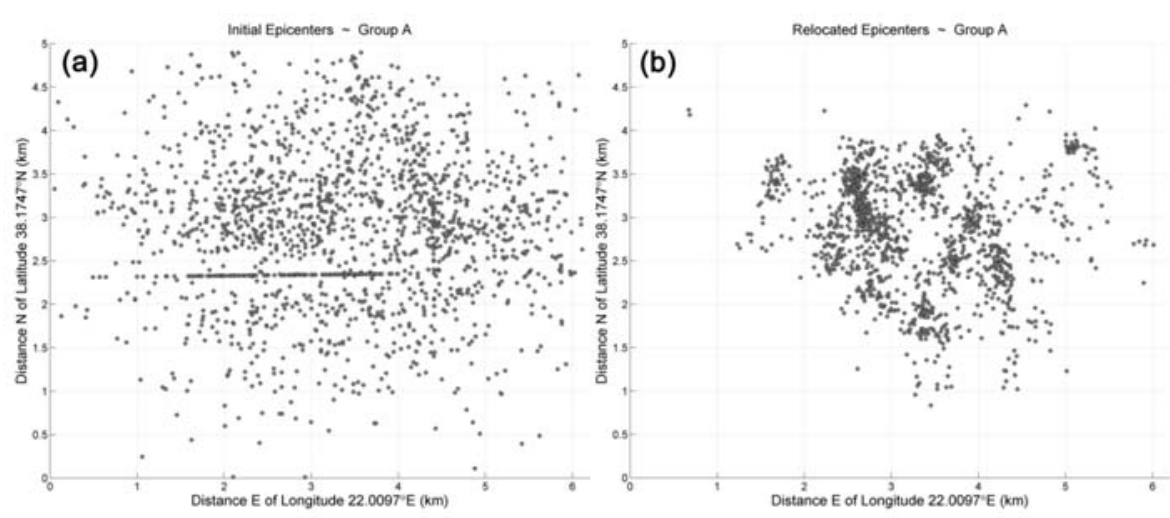

(c)

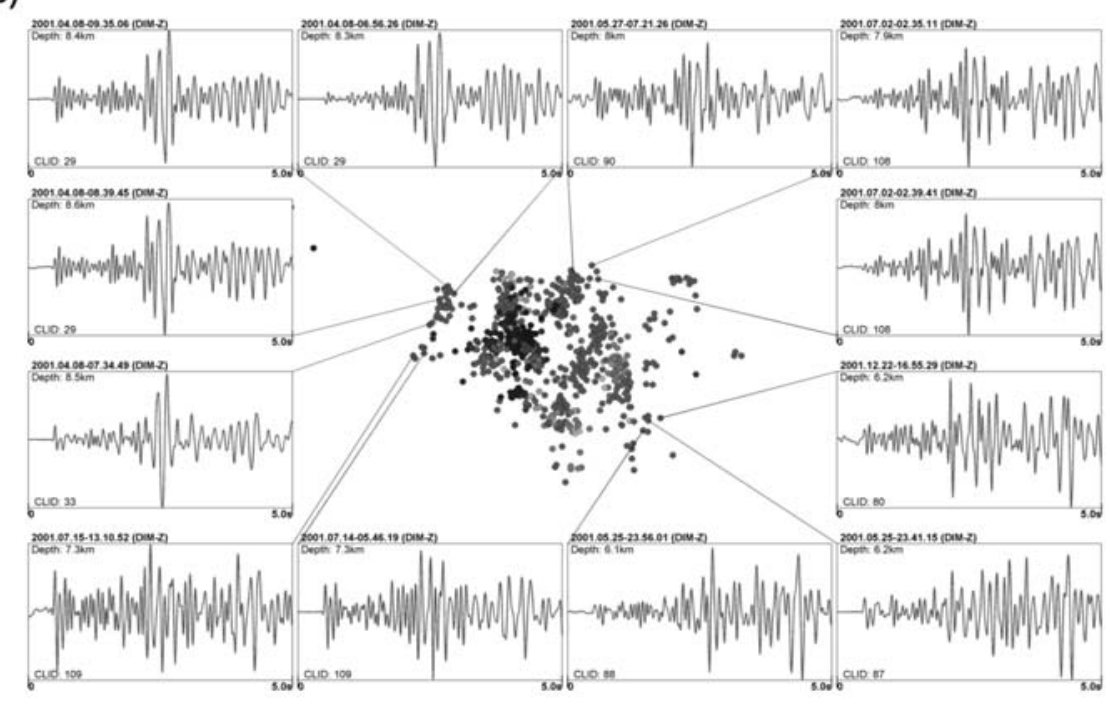

Fig. 6: (a): initial locations of earthquakes in Group A; (b): relocated epicenters using the double-difference method; (c): seismic recordings of the vertical component of station DIM for certain events of Group A. CLID number is the Cluster-ID to which each event belongs. Waveform recordings of events with the same Cluster-ID are similar.

This method could also be expanded to correct other parts of the catalogue by increasing the number of master events, or even be applied for the improvement/addition of arrival-times in events of previous years, since temporal separation poses no limitation to multiplets. The improved catalogue, alongside with cross-correlation differential travel-times were used as input data to a double-difference procedure implemented in the HypoDD software (Waldhauser, 2001). A total of about 5700 events from years 2000-2001 were relocated, 1357 from group A (Fig. 6) and 1449 from group B. From the grouped events of years 2005-2006 a total of 1170 events from year 2005 were relocated (group C) as well as 3508 events from groups D, E and F of year 2006.

\section{References}

Aki, K. \& Richards, P. G. 1980. Quantitative Seismology, Freeman, San Francisco, 2nd edition, 1980. 
Bernard, P., Lyon-Caen, H., Briole, P., Deschamps, A., Boudin, F., Makropoulos, K., Papadimitriou, P., Lemeille, F., Patau, G., Billiris, H., Paradissis, D., Papazissi, K., Castarède, H., Charade, O., Nercessian, A., Avallone, A., Pacchiani, F., Zahradnik, J., Sacks, S. \& Linde, A. 2006. Seismicity, deformation and seismic hazard in the western rift of Corinth: New insights from the Corinth Rift Laboratory (CRL), Tectonophysics 426, 7-30, 2006.

Deichmann, N., \& Garcia-Fernandez, M. 1992. Rupture geometry from high-precision relative hypocenter locations of microearthquake clusters, Geophys. J. Int. 110, 501-517.

Got, J.-L., Frechet, J. \& Klein, F.W. 1994. Deep fault plane geometry inferred from multiplet relative location beneath the south flank of Kilauea. J. Geophys. Res. 99, 15375-15386.

Hansen, S.E., Schwartz, S.Y., DeShon, H.R. \& Gonzalez, V., 2006. Earthquake Relocation and Focal Mechanism Determination Using Waveform Cross Correlation, Nicoya Peninsula, Costa Rica. Bulletin of the Seismological Society of America, Vol. 96, No. 3, pp. 1003-1011.

Janský J., Zahradník J., Sokos E., Serpetsidaki A. \& Tselentis G.A. 2004. Relocation of the 2001 Earthquake Sequence in Aegion, Greece, Studia Geophysica et Geodaetica, Volume 48, Number 2, April 2004, pp. 331-344(14).

Kilb, D. \& Rubin, A.M. 2002. Implications of diverse fault orientations imaged in relocated aftershocks of the Mount Lewis, ML 5.7, California, earthquake. J. Geophys. Res. Vol. 107, No. B11, 2294, doi:10.1029/2001JB000149, 2002.

Lyon-Caen, H., Papadimitriou, P., Deschamps, A., Bernard, P., Makropoulos, K., Pacchiani, F. \& Patau, G. 2004. First results of the CRLN seismic network in the western Corinth Rift: evidence for old-fault reactivation. C. R. Geoscience 336 (2004) 343-351, Tectonics.

Poupinet, G., Ellsworth, V. L. \& Frechet, J. 1984. Monitoring velocity variations in the crust using earthquake doublets: An application to the Calaveras fault, California, Journal of Geophysical Research, Volume 89, Issue B7, p. 5719-5732.

Rubin, A.M., Gillard, D., Got, J.-L. 1999. Streaks of microearthquakes along creeping faults, Nature 400,635-641.

Schaff, D.P., Bokelmann, G.H.R, Ellsworth, W.L., Zanzerkia, E., Waldhauser, F. \& Beroza, G.C. 2004. Optimizing Correlation Techniques for Improved Earthquake Location, Bulletin of the Seismological Society of America, Vol. 94, No. 2, pp. 705-721, April 2004.

Schaff, D.P. \& Waldhauser, F. 2005. Waveform Cross-Correlation-Based Differential Travel-Time Measurements at the Northern California Seismic Network. Bulletin of the Seismological Society of America, Vol. 95, No. 6, pp. 2446-2461.

Waldhauser, F., Ellsworth, W.L. \& Cole, A. 1999. Slip-Parallel Seismic Lineations on the Northern Hayward Fault, California. Geoph. Res. Let. 26, pp. 3525-3528.

Waldhauser, F. \& Ellsworth, W.L. 2000. A double-difference earthquake location algorithm: Method and application to the northern Hayward fault, Bull. Seismol. Soc. Am., 90, 1353-1368, 2000.

Waldhauser, F. 2001. HypoDD: A computer program to compute double-difference earthquake locations, U.S. Geol. Surv. open-file report, 01-113, Menlo Park, California, 2001.

Zahradnik, J., Jansky, J., Sokos, E., Serpetsidaki, A., Lyon-Caen, H. \& Papadimitriou, P. 2004 . Modeling the ML4.7 mainshock of the February-July 2001 earthquake sequence in Aegion, Greece. Journal of Seismology 8: 247-257, 2004.

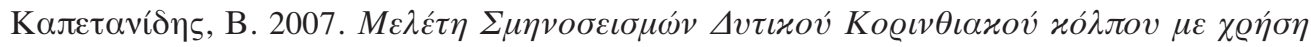

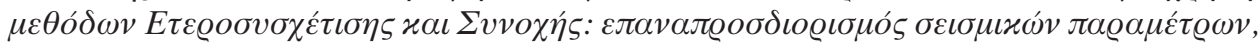

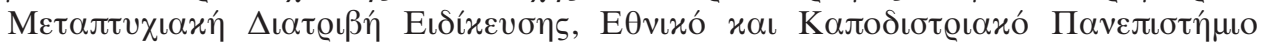

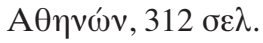

